\title{
Comparative analysis of DNA methylation changes in two rice genotypes under salt stress and subsequent recovery
}

\author{
Wensheng Wang ${ }^{1}$, Fei Huang ${ }^{1}$, Qiao Qin ${ }^{1}$, Xiuqin Zhao ${ }^{1}$, Zhikang $\mathrm{Li}^{1,2}$ and Binying $\mathrm{Fu}^{1,2 *}$ \\ ${ }^{1}$ Institute of Crop Sciences / National Key Facility for Crop Gene Resources and Genetic \\ Improvement, Chinese Academy of Agricultural Sciences, Beijing 100081, China; E-Mails: \\ wsh-wang@163.com (W.W.); vickyhuangfei@163.com (F.H.); tsinemail@163.com (Q.Q.); \\ zhaoxiuqin@caas.cn (X.Z.); lizhikang@caas.cn (Z.L.) \\ ${ }^{2}$ Shenzhen Institute for Innovative Breeding, Chinese Academy of Agricultural Sciences, \\ Shenzhen 518120, China.
}

* Author to whom correspondence should be addressed; E-Mail: fubinying@ caas.cn Tel.: +86-10-82106698; Fax: +86-10-82108559 


\begin{abstract}
DNA methylation, which is one of the best understood epigenetic phenomena, plays an important role in plant responses to environmental stimuli. The rice introgression line IL177-103 and its recurrent parent IR64, which show contrasting salt stress tolerance, were used to characterize DNA methylation changes under salt stress and subsequent recovery using methylation-sensitive amplified polymorphism (MSAP) analysis. The introgression line IL177-103 showed significantly improved tolerance to salinity, as represented by higher relative water content, endogenous abscisic acid content, activity of reactive oxygen species scavenging enzymes, and lower $\mathrm{Na}^{+}$concentration in shoots, compared with IR64. The MSAP results showed that less than $10.5 \%$ of detected DNA methylation sites were genotype specific, in line with their similar genetic background. Salt-induced DNA methylation changes in both genotypes were mostly detected in roots, and the major portion of the salt-induced DNA demethylation/methylation alterations remained even after recovery, implying their inheritance in the present generation. Furthermore, a few sites with stable DNA methylation differences were identified between salt-tolerant IL177-103 and salt-sensitive IR64, thus providing genotype-specific epigenetic markers. Collectively, these results provide valuable data for further dissection of the molecular mechanisms of salt-stress response and tolerance in rice.
\end{abstract}

Keywords: DNA methylation; salt tolerance; epigenetic marker; rice 


\section{Introduction}

Salt stress is a severe environmental factor that limits crop productivity and agricultural sustainability in arid and semi-arid regions of the world [1]. Rice is the staple food crop in many parts of the world, and rice plants are sensitive to salt stress at a young seedling stage as well as at the reproductive stage [2]. Developing salt-tolerant rice cultivars by molecular breeding and biotechnology is an efficient approach to reduce the yield loss caused by salt stress and contribute to food security. Rice genotypes vary considerably in salt-stress tolerance. Genetic studies have revealed that a number of quantitative trait loci are associated with salt tolerance in rice $[3,4]$. However, few studies have investigated the relationship between epigenetics and the molecular mechanisms of salt stress tolerance in rice.

DNA methylation, an important epigenetic mechanism, is involved in the control of all genetic functions, including transcription, post-transcription, DNA repair, gene transposition, and cell differentiation, without an alteration to the DNA sequence $[5,6]$. DNA methylation in plants is well known to be genotype, species, tissue, and developmental stage specific $[7,8]$. In rice, about $8 \%$ of active genes are methylated within their promoter regions and $31 \%$ of expressed genes are methylated in the transcribed regions [9]. More than one-third of all Arabidopsis genes experience a degree of methylation within transcription regions and only 5\% of genes show methylation within promoter regions; methylation of the latter regions is correlated with differential gene expression in a temporal or spatial manner [10].

Increasing evidence indicates that DNA methylation plays a crucial role in regulating gene expression in plant responses to environmental stresses [11-14]. The global DNA methylation levels of a salinity-tolerant wheat cultivar are reduced by exposure to salinity stress and the alteration of DNA methylation in promoter regions may affect gene expression [15]. Salt, cold, and oxidative stresses induce DNA demethylation in the coding sequence of the Nicotiana tabacum glycerophosphodiesterase-like protein gene $(N t G P D L)$ in tobacco leaves, and this demethylation is positively correlated with $N t G D P L$ gene expression [16]. In rice, the gene body methylation may perform an important role in regulating gene expression in an organ- and genotype-specific manner under salinity stress [17]. Furthermore, whole methylome sequencing of Arabidopsis grown in high-salinity soil has revealed that the salt-stressed lineages accumulate $\sim 45 \%$ more differentially methylated 
cytosines at CG sites than the controls [18].

Recently, it has been shown that plants can remember past environmental stimuli through epigenetic memories and can use these memories to aid responses when these events recur [19]. Ding et al. [20] reported that multiple exposures to drought stress enable plants to respond to a new stress by making more rapid adaptive changes to gene expression patterns compared with plants not previously exposed to drought stress. $75 \%$ of differentially methylated cytosine positions induced by salinity stress are inherited, although some may be lost in subsequent generations [18]. However, detailed information on the general pattern of DNA methylation linked with the response of rice to salinity and subsequent recovery, salinity-induced epigenetic memories, and their relationship with salinity tolerance in rice remain to be elucidated.

In this study, two rice genotypes, the salinity-tolerant rice introgression line IL177-103 and its salinity-sensitive recurrent parent IR64, were used to characterize the DNA methylation changes under salt stress and subsequent recovery by methylation-sensitive amplified polymorphism (MSAP) analysis. The genotype- and tissue-specific DNA methylation variations induced by salt stress and a portion of these salt-induced DNA methylation/demethylation sites remain stable during recovery. The present findings provide useful epigenetic information for further molecular dissection of salt stress tolerance in rice.

\section{Materials and Methods}

\subsection{Plant Materials and Growth Conditions}

Two rice lines, IL177-103 and IR64, were used in this study. IR64 is a salinity-sensitive cultivar and has been widely grown in many Asian countries. IL177-103 is a salinity-tolerant $\mathrm{F}_{7}$ line derived from a cross between two ST IR64 introgression lines. SSR markers distributed throughout the rice genome were used to analyze genetic differences between the two genotypes. It was established that IL177-103 differed from IR64 in six genomic segments inherited from two donors, Madhukar and Binam (Figure S1).

Rice seeds were surface sterilized in $0.1 \% \mathrm{NaClO}(\mathrm{v} / \mathrm{v})$ and rinsed several times before germination at $37{ }^{\circ} \mathrm{C}$ in an incubator. After 3 days, germinated seeds were transferred to a 20-L tank filled with Yoshida nutrient solution. The seedlings were cultured for 12 days after germination. Salinity treatment was applied by addition of 
$\mathrm{NaCl}$ to achieve a final concentration of $125 \mathrm{mM}$. After treatment for 4 days, the stressed plants were recovered for 5 days by culture in Yoshida nutrient solution lacking $\mathrm{NaCl}$. The seedlings were grown in a phytotron with $29 / 22^{\circ} \mathrm{C}$ (day/night) temperatures and minimum relative humidity of $70 \%$. The $\mathrm{pH}$ of the nutrient solution was adjusted daily to 5.5 by adding sulfuric acid. The nutrient solution was refreshed weekly.

\subsection{Phenotypic and Physiological Trait Evaluation}

After treatment for 4 days, phenotypic and physiological traits of IL177-103 and IR64 were characterized under control and salt-stress conditions. Shoot length $(\mathrm{cm})$ was measured for each plant. The fresh weight of the roots and shoots was measured before oven drying at $70-75^{\circ} \mathrm{C}$ for $72 \mathrm{~h}$ and the total biomass was determined. Relative water content of each plant was calculated as (FW-DW)/(TW-DW)*100, where FW, DW, and TW arethe fresh weight, dry weight, and total weight of each plant [21]. Sodium and potassium concentrations and the sodium: potassium ratio in shoots were measuredin accordance with the method described by Zang et al. [22].

Leaf tissue from the well-cultured (control) and salinity-stressed plants were used to analyze the endogenous ABA content and activity of antioxidant enzymes. Endogenous ABA content was measured using the plant abscisic acid (ABA) ELISA Kit (Catalog no. CSB-E09159P1). Activity of the antioxidant enzymes SOD, POD, and CAT were determined following previously reported methods [23].

\subsection{Methylation-sensitive Amplification Polymorphism Analysis}

Leaf and root tissue from three replicates were collected from the well-cultured (control), salinity-stressed, and recovered plants at the seedling stage. Genomic DNA of both IL177-103 and IR64 samples were isolated using the improved CTAB method. The MSAP analysis was performed as described by Wang et al. [8]. Three biological replicates for the control and salinity-stressed samples and two biological replicates for the recovered samples were used for the MSAP analysis.

\subsection{Cloning and Characterization of the Differentially Amplified DNA Fragments}

Selected differentially amplified fragments were isolated, reamplified, and purified with Wizard SV Gel and PCR Clean-Up System (Promega, Madison, WI, USA). The reamplified DNA fragments were purified and cloned using the T-vector (Takara, Dalian, China) for sequencing. The sequences obtained were analyzed 
against sequences in the National Center for Biotechnology Information databases using the BLAST tool (http://blast.ncbi.nlm.nih.gov/Blast.cgi).

\section{Results}

\subsection{Phenotypical and PhysiologicalCharacterization of IL177-103 and IR64 under}

\section{Salt Stress}

Under the non-stressed condition no notable phenotypical difference was observed between the IL177-103 and IR64 genotypes at the seedling stage (Table 1). Growth parameters for the two genotypes were evaluated after salt stress treatment. A significantly negative effect on growth parameters including relative water content, shoot length, and total biomass was observed in both genotypes after stress treatment. These parameters were more strongly inhibited for IR64 compared with those of IL177-103 under the saline condition. The $\mathrm{Na}^{+}$concentration and $\mathrm{Na}^{+}: \mathrm{K}^{+}$ratio were significantly increased in shoots of both genotypes under salt stress. The increase in these two parameters was more evident in IR64 (Table 1), which indicated that shoots of IL177-103 suffered less severe $\mathrm{Na}^{+}$toxicity.

To investigate physiological changes in response to salt stress for each genotype, endogenous abscisic acid (ABA) content and activity of antioxidant enzymes in the leaf were measured. Compared with the control condition, both genotypes exhibited higher endogenous ABA content and activities of catalase (CAT), peroxidase (POD), and superoxide dismutase (SOD) under salinity treatment (Figure1). The endogenous ABA content and activity of CAT in IL177-103 were significantly higher than those of IR64 (Figure 1A and 2B) after salinity stress for 4 days. However, the activities of POD and SOD did not show significant differences between IL177-103 and IR64 (Figure 1C and 1D). These results indicated that the endogenous ABA content and CAT might play important roles in the salinity tolerance of IL177-103.

\subsection{General DNA Methylation Patterns in IL177-103 and IR64 under Different}

\section{Treatments}

Forty-three to 50 primer combinations were used for the MSAP analysis, with which 694-826 clear and reproducible fragments were amplified in leaf or root 
samples of IL177-103 and IR64 growing under control, salt stress, and subsequent recovery conditions. Tables 2 and 3 summarize the general cytosine methylation patterns in the leaves and roots of each genotype under the three conditions. Based on the MSAP profiles, the number of methylated (hemi- and fully methylated) DNA bands was determined. A total of 193-236 bands (Type II + Type III + Type IV), accounting for $26.5-28.7 \%$ of the total bands, were polymorphic among the samples. The total DNA methylation levels in leaves of both genotypes were similar (Table 2). However, when measured by the number and percentage of total fully-methylated bands in roots under the three conditions, for both genotypes the general pattern was control > stress $>$ recovery (Table 3$)$.

A comparative analysis of the DNA methylation patterns in leaves or roots of both genotypes under each condition was performed. As shown in Supplemental Table S2, the major portion of the detected bands was common to IL177-103 and IR64. A total of $6.79-10.42 \%$ of the bands were genotype specific, e.g. 51/47 and 52/48 bands (Type I+II+III) were specifically amplified by MSAP in leaves of IL177-103/IR64 under control and salt stress conditions, respectively. In contrast, 73/61 and 85/70 bands (Type I+II+III) were detected in roots of IL177-103/IR64 under control and salt stress conditions, respectively. A slightly higher number of genotype-specific bands were detected for IL177-103 than for IR64.

\subsection{DNA Methylation Changes in IL177-103 and IR64 under Salt Stress and}

\section{Subsequent Recovery}

The DNA methylation alterations for both genotypes under salt stress and subsequent recovery were further comparatively analyzed. Salt stress resulted in only slight changes in DNA methylation in leaves of both genotypes; only four DNA bands were identified as methylated/demethylated under the stress or recovery conditions (Table 4). However, significant changes in DNA methylation were observed in roots of both genotypes under salt stress and subsequent recovery (Table 4). The same number of demethylated DNA bands (22 bands in each genotype; classes $\mathbf{a}+\mathbf{b}$ ) was detected in both genotypes under salt stress; these bands were remethylated (class a) or remained hypomethylated (class b) on recovery. In addition, a few bands (4 and 7 in IL177-103 and IR64, respectively) were methylated under salt stress, but demethylated (class c) or remained methylated (class d) under subsequent recovery. 
Collectively, these results indicated that salt-stress-induced DNA methylation changes were largely root specific, and mostly involved demethylation; in addition, the major portion (classes $\mathbf{b}$ and $\mathbf{d}$ ) of these DNA methylation changes (demethylation /methylation) were stable in the recovery condition (after the stress was removed).

\subsection{Epigenetic Alleles between IL177-103 and IR64}

Four MSAP fragments showed stable DNA methylation polymorphism between salinity-tolerant IL177-103 and salinity-sensitive IR64 under the control, stress and recovery conditions (Table 5 and Figure S1). The fragments V1 and V2 were hypomethylated in IR64 under the control, salinity, and recovery conditions, but in IL177-103 both fragments were hypermethylated under the three conditions. The fragments V3 and V4 were hypermethylated in IR64 but hypomethylated in IL177-103. Two possible reasons for this polymorphism are differences at the genetic level (DNA sequences) between IR64 and IL177-103, and differences at the epigenetic level (DNA methylation patterns, i.e., epigenetic alleles). To validate the observed polymorphism, two experiments were performed. First, we sequenced the four fragments, and based on the BLAST results the four fragments were located on chromosomes 2, 3, and 7, respectively (Table 5). However, no differences were observed between IL177-103 and IR64 based on a survey of 385 simple sequence repeat (SSR) markers distributed across the rice genome (Figure S2). Second, we designed sequence-specific PCR primers based on the four fragment sequences and sequenced the PCR products. No differences in DNA sequence between IL177-103 and IR64 were observed within the four regions. Those two results indicated that DNA methylation differences may be responsible for the polymorphisms between IL177-103 and IR64. These stable DNA methylation differences (epigenetic alleles) between cultivars that show different salinity tolerance may be candidate epigenetic markers, which previously have been reported to be potentially linked to phenotypic variations between closely related strains [24].

\subsection{BLAST Results and Methylation Patterns of Polymorphic Methylated DNA}

\section{Fragments}

A random set of 19 polymorphic methylated fragments (four from leaf tissue and 15 from root tissue) were cloned and sequenced. The sequences of the cloned bands 
showed an average length of $173 \mathrm{bp}$, ranging from 62 to $602 \mathrm{bp}$ (Table 6). The fragments were distributed widely in the rice genome except on chromosomes 7, 9, 11, and 12, indicating that salinity induced genome-wide alteration in DNA methylation/demethylation.

The methylation patterns of the cloned fragments from leaf tissue are summarized in Table 6. Two fragments (L1 and L2) belonged to class b (demethylated during salinity stress, and remained hypomethylated after recovery). The other two fragments (L3 and L4) belonged to class e (DNA methylation pattern remained unchanged under salinity stress, but changed after recovery). Based on the BLAST results, the four fragments were homologous to genes encoding a receptor-like protein kinase, mitochondrial-processing peptidase, and Myb-like transcription factor.

The methylation pattern in root tissue induced by salinity was more complicated than that of leaf tissue. Eight fragments belonged to class $\mathbf{b}$, two fragments belonged to class $\mathbf{e}$, one fragment belonged to class $\mathbf{d}$ (methylated during salinity stress, and remained methylated after recovery), three fragments belonged to class a (demethylated during salinity stress, but remethylated after recovery), and one fragment belonged to class $\mathbf{c}$ (methylated during salinity stress, but demethylated after recovery). Among the 15 polymorphic methylated DNA fragments analyzed from root tissue, four encoded reverse transcriptase, DNA polymerase, ATPase, and hexokinase. Six fragments were highly homologous to ribosomal protein, RING-H2 finger protein, thaumatin, basic $7 \mathrm{~S}$ globulin, and histone-like transcription factor. The remaining fragments were homologous to genes encoding DUF domains, transposon protein, hypothetical proteins, and cyclic nucleotide-gated ion channel. These results indicate that the salinity-induced methylation/demethylation bands detected involved genes with a wide range of functions, including those associated with stress responsiveness.

\section{Discussion}

Epigenetic mechanisms such as DNA methylation play a key role in plant response to environmental stresses by adjusting physiological and developmental mechanisms through differentially regulating genome-wide gene expression [25-27]. Demethylation usually leads to transcriptional activation of certain functionally inactive genes when plants are exposed to abiotic stresses [28]. The present results 
showed that salinity induced genome-wide changes in the DNA methylation status of the salinity-tolerant rice introgression line IL177-103 and salinity-sensitive cultivar IR64. In particular, salinity tended to reduce the overall DNA methylation levels in root tissue of both genotypes. These results are consistent with previous reports that showed environmental stimuli, such as drought, salinity, cold, heat, and heavy metals, tend to cause hypomethylation of the genomic DNA or stress-response genes [8,14,16,29-31]. Tissue-specific alterations were observed among the salinity-induced DNA methylation changes in rice. In root tissue, salinity stress dramatically decreased the methylation level, but in leaf tissue only slight changes in methylation level were observed in this study. Tissue-specific gene expression is known to be controlled by DNA methylation [32]. Differences in DNA methylation changes in roots and leaves are indicative of the unique biological functions of rice roots and leaves in response to salinity stress. The possible reason for the tissue-specific DNA methylation changes may be because of the fact that root tissue experiences the stress first, and is in direct contact with the surrounding high-salinity environment. Hypomethylation in roots suggests the preparedness of more stress-responsive genes to respond to salinity stress $[17,32]$. The present results suggest that tissue-specific DNA methylation changes may be one of the epigenetic mechanisms involved in rice adaptation to salinity stress.

Increasing evidence indicates that epigenetic mechanisms such as DNA methylation are essential for stress memories and adaptation in plants [18,20,33]. For example, heat stress causes epigenomic and physiological changes in Arabidopsis thaliana, and these changes may be transmitted to progeny partially reliant on DCL2 and DCL3 [34]. Transgenerational epigenetic inheritance may be the key reason for the transgenerational phenotypic changes induced by heat and salt stress in Arabidopsis thaliana [35]. In the current study, two major types of salinity-induced methylation changes in root tissue were identified. The first type, including classesa and c, consisted of demethylation/methylation under salt stress, but reversal to the original status after 5 days recovery. The second type, including classes $\mathbf{b}$ and $\mathbf{d}$, comprised DNA hypomethylation/hypermethylation changes that persisted even after subsequent recovery (Table 4). The second type of salinity-induced DNA methylation changes has the capacity to 'remember' the environmental stress and maintain the stress-induced epigenetic states, and to be inherited through mitotic cell divisions in the present generation. However, whether these salt-induced DNA methylation alterations can be transmitted to the next generation needs further study. 
Four epigenetic alleles showed stable different DNA methylation states between IL177-103 and IR64, which differ in salinity tolerance. These DNA methylation differences (epigenetic alleles) between the two genotypes may be candidate epigenetic markers, which previously have been reported to be potentially linked to phenotypic variation between closely related strains. Epialleles might cause phenotypic differences between cultivars with a genetically close relationship. Identification of epialleles underlying natural variation will be an important issue in future rice breeding [24,36]. Epigenetic alleles may be involved in diverse phenomenaranging from development to genome evolution and defense against transposons[37]. Zhang et al. [38] identified a gain-of-function epiallele (Epi-df) of rice FIE1; this allele caused a dwarf stature and various floral defects. In plants, commonly encountered natural environmental stresses can accelerate the accumulation and change the profiles of novel inherited variants [18]. During long-term interactions between plants and environmental stresses, salinity-tolerant and -sensitive genotypes may form distinct fixed methylation patterns in some genomic or gene regions. These distinct methylation patterns might be linked to stress tolerances and be inherited in multiple subsequent generations.

\section{Acknowledgements}

We would like to thank the National Natural Science Foundation of China (31271694), the Bill \& Melinda Gates Foundation (OPPGD1393), the International S\&T Cooperation Program of China (2012DFB32280), the Shenzhen Peacock Plan (20130415095710361) and the CAAS Innovative Team Award for their financial supports. 


\section{References}

1. G. Ondrasek, Z. Rengel, S. Veres, Soil salinisation and salt stress in crop production. In Shanker AK and Venkateswarlu B, eds, Abiotic Stress in Plants: Mechanisms and Adaptations, ISBN 978-953-307-394-1. InTech, 2011, pp, 171-190.

2. S. Lutts, J.M. Kinet, J. Bouharmont, Changes in plant response to $\mathrm{NaCl}$ during development of rice (Oryza sativa L.) varieties differing in salinity resistance. J. Exp. Bot. 46 (1995) 1843-1852.

3. R.K. Singh, G.B. Gregorio, R.K. Jain, QTL mapping for salinity tolerance in rice. Physiol. Mol. Biol. Plants 13 (2007) 87-99.

4. M.J. Thomson,; M. de Ocampo, J. Egdane, et al., Characterizing the Saltol quantitative trait locus for salinity tolerance in rice. Rice 3 (2010) 148-160.

5. K.J.F. Verhoeven, J.J. Jansen, Dijk van, et al., Stress-induced DNA methylation changes and their heritability in asexual dandelions. New Phytol. 185 (2010) $1108-1118$.

6. B.F. Vanyushin, V.V. Ashapkin, DNA methylation in higher plants: past, present and future. Biochimica et biophysica acta 1809 (2011) 360-368.

7. J.A. Law, S.E. Jacobsen, Molecular biology. Dynamic DNA methylation. Science 323 (2009) 1568-1569.

8. W.S. Wang, Y.J. Pan, X.Q.Zhao, et al., Drought-induced site-specific DNA methylation and its association with drought tolerance in rice (Oryza sativa L.). J. Exp. Bot. 62 (2011) 1951-1960.

9. H.H. Yan, S. Kikuchi, P. Neumann, et al., Genome-wide mapping of cytosine methylation revealed dynamic DNA methylation patterns associated with genes and centromeres in rice. Plant J. 63 (2010) 353-365.

10. D. Zilberman, M. Gehring, R.K. Tran, et al., Genome-wide analysis of Arabidopsis thaliana DNA methylation uncovers an inter dependence between methylation and transcription. Nat. Genet. 39 (2007) 61-69.

11. C.A. Cullis, Mechanisms and control of rapid genomic changes in flax. Ann. Bot. 95 (2005) 201-206.

12. A. Boyko, P. Kathiria, F.J. Zemp, et al., Transgenerational changes in the genome stability and methylation in pathogen-infected plants: (Virus-induced plant genome instability). Nucleic Acids Res. 35 (2007) 1714-1725. 
13. A. Boyko, I. Kovalchuk, Epigenetic control of plant stress response. Environ. Mol. Mutagen. 49 (2008) 61-72.

14. W.S. Wang, X.Q. Zhao, Y.J. Pan, et al., DNA methylation changes detected by methylation-sensitive amplified polymorphism in two contrasting rice genotypes under salt stress. J. Genet. Genomics 38 (2011) 419-424.

15. M. Wang, L.M. Qin, C. Xie, et al., Induced and constitutive DNA methylation in a salinity-tolerant wheat introgression line. Plant Cell Physiol. 55 (2014) 1354-1365.

16. C.S. Choi, H. Sano, Abiotic-stress induces demethylation and transcriptional activation of a gene encoding a glycerophosphodiesterase-like protein in tobacco plants. Mol. Genet. Genomics 277 (2007) 589-600.

17. R. Karan, T.Deleon, H. Biradar, et al., Salt stress induced variation in DNA methylation pattern and its influence on gene expression in contrasting Rice genotypes. PLoS ONE 7 (2012) e40203.

18. C.F. Jiang, A. Mithani, E.J. Belfield, et al., Environmentally responsive genome-wide accumulation of de novo Arabidopsis thaliana mutations and epimutations. Genome Res. 24 (2014) 1821-1829.

19. T. Kinoshita, M. Seki, Epigenetic memory for stress response and adaptation in plants. Plant Cell Physiol. 55 (2014) 1859-1863

20. Y. Ding, M.Fromm, Z. Avramova, Multiple exposures to drought 'train' transcriptional responses in Arabidopsis. Nat. Commun. 3 (2012) 740.

21. A. Larbi, A. Mekliche, Relative water content (RWC) and leaf senescence as screening tools for drought tolerance in wheat. In Cantero-Martínez C, Gabiña D, eds. Mediterranean Rainfed Agriculture: Strategies for Sustainability. Zaragoza: CIHEAM, 2004 193-196.

22. J.P. Zang, Y.Sun, Y. Wang, et al., Dissection of genetic overlap of salt tolerance QTLs at the seedling and tillering stages using backcross introgression lines in rice. Sci. China Ser C-Life Sci. 51 (2008) 583-591.

23. V. Bonnecarrère, O. Borsani, P. Díaz, et al., Response to photoxidative stress induced by cold in japonica rice is genotype dependent. Plant Sci. 180 (2011) $726-732$.

24. M. Takata, K.Yuji, S. Yoshio, DNA methylation polymorphisms in rice and wild rice strains: detection of epigenetic markers. Breeding Sci. 55 (2005) 57-63.

25. L. López-Maury, S. Marguerat, J. Bähler, Tuning gene expression to changing 
environments: from rapid responses to evolutionary adaptation. Nat. Rev. Genet. 9 (2008) 583-93.

26. Y.L. Lu, T.Z.Rong, M.J. Cao, Analysis of DNA methylation in different maize tissues. J. Genet. Genomics 35 (2008) 41-48.

27. B. Angers, E.Castonguay, R. Massicotte, Environmentally induced phenotypes and DNA methylation: how to deal with unpredictable conditions until the next generation and after. Mol. Eco. 19 (2010) 1283-1295.

28. A.P. Santos, L.Ferreira, J. Maroco, et al., Abiotic stress and induced DNA hypomethylation cause interphase chromatin structural changes in rice rDNA loci. Cytogene. Genome Res. 132 (2011) 297-303.

29. R. Alina, S.Sgorbati, A.Santagostino, et al., Specific hypomethylation of DNA is induced by heavy metals in white clover and industrial hemp. Physiol. Plantarum 121 (2004) 472-480.

30. Y.J. Pan, W.S. Wang, X.Q Zhao, et al., DNA methylation alterations of rice in response to cold stress. Plant Omics J. 7 (2011) 364-369.

31. D.H. Sanchez, J.Paszkowski, Heat-induced release of epigenetic silencing reveals the concealed role of an imprinted plant gene. PLoS Genet. 10 (2014) e1004806.

32. X. Gao, D.H.Cao, J.Liu, et al., Tissue-specific and cation/anion-specific DNA methylation variations occurred in $C$. virgata in response to salinity stress. PLoS ONE 8 (2013) e78426.

33. Y. Kinoshita, H. Saze, T. Kinoshita, et al., Control of $F W A$ gene silencing in Arabidopsis thaliana by SINE-related direct repeats. Plant J. 49 (2007) 38-45.

34. Z. Migicovsky, Y.L. Yao, I. Kovalchuk, Transgenerational phenotypic and epigenetic changes in response to heat stress in Arabidopsis thaliana. Plant Signal. Behav. 9 (2014) e27971.

35. L. Suter, A. Windmer, Environmental heat and salt stress induce transgenerational phenotypic changes in Arabidopsis thaliana. PLoS ONE 8 (2013) e60364.

36. H. Peng, G.H. Jiang, J. Zhang, et al., DNA methylation polymorphism and stability in Chinese indica hybrid rice. Sci. China Life Sci. 56 (2013) 1097-1106.

37. T. Kakutani, Epi-alleles in plants: Inheritance of epigenetic information over generations. Plant Cell Physiol. 43 (2002) 1106-1111.

38. L.G. Zhang, Z.J.Cheng, R.Z. Qin, et al., Identification and characterization of an epi-allele of FIE1 reveals a regulatory linkage between two epigenetic marks in rice. Plant Cell 24 (2012) 4407-21. 


\section{Table legends:}

Table 1.Morphological and physiological traits of IR64 and IL177-103 under control and salinity conditions

Table 2. DNA methylation changes in leaves of IL177-103 and IR64 under salt stress and subsequent recovery

Table 3. DNA methylation changes in roots of IL177-103 and IR64 under salt stress and subsequent recovery

Table 4. Summary of DNA methylation pattern changes of IL177-103 and IR64 under salt stress and subsequent recovery

Table 5. BLAST results and DNA methylation patterns ofepigenetic alleles between IR64 and IL177-103

Table 6. BLAST results and methylation patterns of a randomly selected polymorphic methylated DNA fragments

\section{Figure legends:}

Figure1.Endogenous ABA content and activity of antioxidant enzymes in seedlings of the two rice genotypes under control(CK) and salinity conditions.

\section{Supplementary Materials}

Supplementary materials can be found at

Table S1. Adapter and primer sequences used for MSAP analysis

Table S2. Comparative analysis of different MSAP bands in leaves and roots of IL177-103 and IR64 under control, salt stress, and recovery conditions

Figure S1. Genetic composition of IL177-103 at 6 genomic regions (loci) introgressed and pyramided from two different donors, Madhukar and Binam, in the IR64 genetic background based on SSR markers.

Figure S2. DNA methylation patterns in IL177-103 and IR64 under control, salt stress and recovery conditions 
Table 1.Morphological and physiological traits of IR64 and IL177-103 under control and salinity conditions

\begin{tabular}{|c|c|c|c|c|c|c|}
\hline Treatment & Genotype & $\begin{array}{c}\text { Relative } \\
\text { water } \\
\text { content } \\
(\%)\end{array}$ & $\begin{array}{l}\text { Shoot } \\
\text { length } \\
(\mathrm{cm})\end{array}$ & $\begin{array}{c}\text { Total } \\
\text { biomass } \\
\text { (mg/plant) }\end{array}$ & $\begin{array}{c}\text { Shoots } \mathrm{Na}^{+} \\
\text {concentration } \\
\text { (mmol/g dry } \\
\text { weight) }\end{array}$ & $\begin{array}{l}\mathrm{Na}^{+} / \mathrm{K}^{+} \\
\text {ratio in } \\
\text { shoots }\end{array}$ \\
\hline \multirow{2}{*}{ Control } & IR64 & $83.7 \pm 5.1$ & $32.8 \pm 0.1$ & $53.34 \pm 1.8$ & $0.03 \pm 0.01$ & 0.03 \\
\hline & IL177-103 & $82 \pm 3.0$ & $34.5 \pm 0.9$ & $53.83 \pm 2.5$ & $0.04 \pm 0.01$ & 0.03 \\
\hline \multirow{2}{*}{ Salt } & IR64 & $64.5 \pm 3.7$ & $18.0 \pm 0.8$ & $24.93 \pm 1.6$ & $1.31 \pm 0.11$ & 1.63 \\
\hline & IL177-103 & $76.9 \pm 2.1$ & $24.8 \pm 1.0$ & $33.61 \pm 2.2$ & $0.96 \pm 0.05$ & 1.25 \\
\hline
\end{tabular}

Table 2. DNA methylation changes in leaves of IL177-103 and IR64 under salt stress and subsequent recovery

\begin{tabular}{ccccccc}
\hline Genotype & \multicolumn{3}{c}{ IL177-103 } & \multicolumn{3}{c}{ IR64 } \\
\hline MSAP Band Types a) & Control & Salinity & Recovery & Control & Salinity & Recovery \\
I & 500 & 502 & 500 & 499 & 501 & 499 \\
II & 3 & 3 & 3 & 3 & 3 & 3 \\
III & 193 & 193 & 193 & 190 & 190 & 190 \\
IV & 2 & 0 & 2 & 2 & 0 & 2 \\
Total amplified bands & 698 & 698 & 698 & 694 & 694 & 694 \\
Total methylated bands & 198 & 196 & 198 & 195 & 193 & 195 \\
MSAP (\%) & 28.37 & 28.08 & 28.37 & 28.1 & 27.81 & 28.1 \\
\hline Full methylated bands & 195 & 193 & 195 & 192 & 190 & 192 \\
Full methylation ratio (\%) & 27.94 & 27.65 & 27.94 & 27.67 & 27.38 & 27.67 \\
\hline
\end{tabular}

a) Type II = hemi-methylated bands and types III+IV are full methylated bands. Total methylated bands $=$ II $+\mathrm{III}+\mathrm{IV}$

Table 3. DNA methylation changes in roots of IL177-103 and IR64 under salt stress and subsequent recovery

\begin{tabular}{ccccccc}
\hline Genotype & \multicolumn{3}{c}{ IL177-103 } & \multicolumn{3}{c}{ IR64 } \\
\hline MSAP Band Types a) & Control & Stress & Recovery & Control & Stress & Recovery \\
I & 590 & 601 & 605 & 580 & 588 & 598 \\
II & 5 & 7 & 5 & 5 & 7 & 5 \\
III & 205 & 210 & 206 & 203 & 208 & 203 \\
IV & 26 & 8 & 10 & 26 & 11 & 8 \\
Total amplified bands & 826 & 826 & 826 & 814 & 814 & 814 \\
Total methylated bands & 236 & 225 & 221 & 234 & 226 & 216 \\
MSAP (\%) & 28.57 & 27.24 & 26.76 & 28.75 & 27.76 & 26.54 \\
\hline Full methylated bands & 231 & 218 & 216 & 229 & 219 & 211 \\
Full methylation ratio (\%) & 27.97 & 26.39 & 26.15 & 28.13 & 26.90 & 25.92 \\
\hline
\end{tabular}

a) Type II = hemi-methylated bands and types III+IV are full methylated bands. Total methylated bands $=$ II + III + IV 
Table 4. Summary of DNA methylation pattern changes of IL177-103 and IR64 under salt stress and subsequent recovery

\begin{tabular}{ccccc}
\hline \multirow{2}{*}{ Band class * $^{2}$ Leaves } & \multicolumn{2}{c}{ Roots } \\
& IL177-103 & IR64 & IL177-103 & IR64 \\
\hline a & 2 & 2 & 6 & 5 \\
b & 0 & 0 & 16 & 17 \\
c & 0 & 0 & 2 & 4 \\
d & 0 & 0 & 2 & 3 \\
e & 2 & 2 & 4 & 4 \\
f & 694 & 690 & 795 & 781 \\
\hline Total & 698 & 694 & 826 & 814 \\
\hline
\end{tabular}

*a: Demethylated by salinity, but remethylated after recovery; b:Demethylated by salinity, and remaining hypomethylated after recovery; c: Methylated by salinity, but demethylated after recovery; d: Methylated by salinity, and remaining methylated after recovery;e: DNA methylation pattern remained unchanged under salinity, but changed after recovery; h: DNA methylation pattern was unchanged under all three conditions; f: DNA methylation pattern was unchanged under all conditions 
Table 5.BLAST results and DNA methylation patterns ofepigenetic alleles between IR64 and IL177-103

\begin{tabular}{|c|c|c|c|c|c|c|c|c|c|c|c|c|c|c|c|c|c|c|}
\hline \multirow{3}{*}{ Code } & \multirow{3}{*}{$\begin{array}{c}\text { Primer } \\
\text { combination }\end{array}$} & \multirow{3}{*}{$\begin{array}{l}\text { Size } \\
\text { (bp) }\end{array}$} & \multirow{3}{*}{$\begin{array}{c}\text { Accession } \\
\text { No./Locus Name }\end{array}$} & \multicolumn{2}{|c|}{ MSAP Fragments } & \multirow{3}{*}{ Sequence homology } & \multicolumn{6}{|c|}{ IR64 } & \multicolumn{6}{|c|}{ IL177-103 } \\
\hline & & & & \multirow{2}{*}{ Physical position (bp) } & \multirow{2}{*}{$\begin{array}{c}\text { Nucl.id } \\
(\%)\end{array}$} & & \multicolumn{2}{|c|}{ Control } & \multicolumn{2}{|c|}{ Salinity } & \multicolumn{2}{|c|}{ Recovery } & \multicolumn{2}{|c|}{ Control } & \multicolumn{2}{|c|}{ Salinity } & \multicolumn{2}{|c|}{ Recovery } \\
\hline & & & & & & & $\mathbf{E} / \mathbf{H}$ & $\mathbf{E} / \mathbf{M}$ & $\mathbf{E} / \mathbf{H}$ & $\mathbf{E} / \mathbf{M}$ & $\mathbf{E} / \mathbf{H}$ & $\mathbf{E} / \mathbf{M}$ & $\mathbf{E} / \mathbf{H}$ & $\mathbf{E} / \mathbf{M}$ & $\mathbf{E} / \mathbf{H}$ & $\mathbf{E} / \mathbf{M}$ & $\mathbf{E} / \mathbf{H}$ & $\mathbf{E} / \mathbf{M}$ \\
\hline $\mathrm{V} 1$ & H5/M39 & 141 & NC_008400.2 & $7106272-7106386$ & 98 & Oryza sativa Japonica Group DNA, chr 7 & + & + & + & + & + & + & - & - & - & - & - & - \\
\hline V2 & H6/M34 & 308 & LOC_Os03g57660 & $33717128-33717409$ & 99.44 & Peptidase, putative, expressed & + & + & + & + & + & + & - & - & - & - & - & - \\
\hline V3 & H7/M39 & 215 & LOC_Os02g01790 & $434365-434553$ & 100 & Expressed protein & - & - & - & - & - & - & + & + & + & + & + & + \\
\hline V4 & H9/M37 & 286 & LOC_Os02g46510 & 29247359-29247496 & 100 & Adaptor complexes medium subunit family domain & - & - & - & - & - & - & + & + & + & + & + & + \\
\hline
\end{tabular}

“H”: Hpa II; “M”: Msp I; "E”: EcoR I; “+”: Present; “-”: Absent

Table 6.BLAST results and methylation patterns of a randomly selected polymorphic methylated DNA fragments

\begin{tabular}{|c|c|c|c|c|c|c|c|c|c|c|c|c|c|c|c|c|c|c|}
\hline \multirow{3}{*}{ Code } & \multicolumn{6}{|c|}{ MSAP fragments } & \multicolumn{4}{|c|}{ Control } & \multicolumn{4}{|c|}{ Salinity } & \multicolumn{4}{|c|}{ Recovery } \\
\hline & \multirow{2}{*}{$\begin{array}{c}\text { Primer } \\
\text { combination }\end{array}$} & \multirow{2}{*}{ Tissue } & \multirow{2}{*}{$\begin{array}{l}\text { Size } \\
\text { (bp) }\end{array}$} & \multirow{2}{*}{$\begin{array}{c}\text { Accession } \\
\text { No./Locus Name }\end{array}$} & \multirow{2}{*}{ Nucl.id(\%) } & \multirow{2}{*}{ Sequence homology } & \multicolumn{2}{|c|}{ IL177-103 } & \multicolumn{2}{|c|}{ IR64 } & \multicolumn{2}{|c|}{ IL177-103 } & \multicolumn{2}{|c|}{ IR64 } & \multicolumn{2}{|c|}{ IL177-103 } & \multicolumn{2}{|c|}{ IR64 } \\
\hline & & & & & & & $\mathbf{E} / \mathbf{H}$ & $\mathbf{E} / \mathbf{M}$ & $\mathbf{E} / \mathbf{H}$ & $\mathbf{E} / \mathbf{M}$ & $\mathbf{E} / \mathbf{H}$ & $\mathbf{E} / \mathbf{M}$ & $\mathbf{E} / \mathbf{H}$ & $\mathbf{E} / \mathbf{M}$ & $\mathbf{E} / \mathbf{H}$ & $\mathbf{E} / \mathbf{M}$ & $\mathbf{E} / \mathbf{H}$ & $\mathbf{E} / \mathbf{M}$ \\
\hline L1 & H3/M311 & Leaves & 235 & LOC_Os02g06600 & 99 & Receptor-like protein kinase 2 precursor & - & - & - & - & + & + & + & + & + & + & + & + \\
\hline L2 & H6/M34 & Leaves & 116 & NC_008397.2 & 97 & Oryza sativa Japonica Group DNA, chr 4 & - & - & - & - & + & + & + & + & + & + & + & + \\
\hline L3 & H1/M316 & Leaves & 602 & LOC_Os01g09560 & 98 & $\begin{array}{l}\text { Mitochondrial-processing peptidase subunit } \\
\text { alpha }\end{array}$ & + & + & + & + & + & + & + & + & - & - & - & - \\
\hline
\end{tabular}




\begin{tabular}{|c|c|c|c|c|c|c|c|c|c|c|c|c|c|c|c|c|c|c|}
\hline L4 & H1/M316 & Leaves & 128 & LOC_Os06g40710 & 77 & $\begin{array}{l}\text { Myb-like DNA-binding domain containing } \\
\text { protein }\end{array}$ & + & + & + & + & + & + & + & + & - & - & - & - \\
\hline R1 & H8/M312 & Roots & 166 & Os05g0126900 & 86 & $\begin{array}{l}\text { Reverse transcriptase, RNA-dependent } \\
\text { DNA polymerase family protein }\end{array}$ & - & - & - & - & + & + & + & + & + & + & + & + \\
\hline $\mathrm{R} 2$ & H1/M34 & Roots & 62 & Os05g0282900 & 95 & Conserved hypothetical protein & - & - & - & - & + & + & + & + & + & + & + & + \\
\hline R3 & H1/M35 & Roots & 67 & Os02g0627700 & 88 & Cyclic nucleotide-gated ion channel & - & - & - & - & + & + & + & + & + & + & + & + \\
\hline R4 & H7/M35 & Roots & 243 & Os05g0402800 & 92 & $\begin{array}{l}\text { ATPase, P-type, } \\
\mathrm{K} / \mathrm{Mg} / \mathrm{Cd} / \mathrm{Cu} / \mathrm{Zn} / \mathrm{Na} / \mathrm{Ca} / \mathrm{Na} / \mathrm{H} \text {-transporter }\end{array}$ & - & - & - & - & + & + & + & + & + & + & + & + \\
\hline R5 & H10/M38 & Roots & 108 & Os03g0852300 & 81 & $\begin{array}{l}\text { Histone-like transcription factor and } \\
\text { archaeal histone }\end{array}$ & - & - & - & - & + & + & + & + & + & + & + & + \\
\hline R6 & H8/M36 & Roots & 65 & Os02g0241500 & 95 & DUF4216; Domain of unknown function & - & - & - & - & + & + & + & + & + & + & + & + \\
\hline R7 & H1/M34 & Roots & 260 & LOC_Os10g41310 & 70 & $\begin{array}{l}\text { DUF630/DUF632 domains containing } \\
\text { protein }\end{array}$ & - & - & - & - & + & + & + & + & + & + & + & + \\
\hline $\mathrm{R} 8$ & H6/M310 & Roots & 185 & LOC_Os01g33410 & 63 & Transposon protein & - & - & - & - & + & + & + & + & + & + & + & + \\
\hline R9 & H8/M34 & Roots & 168 & Os06g0647100 & 89 & Ribosomal protein L35 & - & - & - & - & - & - & - & - & + & + & + & + \\
\hline $\mathrm{R} 10$ & H9/M313 & Roots & 131 & LOC_Os04g28074 & 67 & Expressed protein & - & - & - & - & - & - & - & - & + & + & + & + \\
\hline R11 & H8/M312 & Roots & 106 & LOC_Os08g34550 & 69 & RING-H2 finger protein & + & + & + & + & - & - & - & - & - & - & - & - \\
\hline R12 & H10/M38 & Roots & 195 & Os04g0689900 & 88 & Thaumatin & - & - & - & - & + & + & + & + & - & - & - & - \\
\hline $\mathrm{R} 13$ & H5/M33 & Roots & 130 & Os07g0197100 & 78 & Hexokinase & - & - & - & - & + & + & + & + & - & - & - & - \\
\hline R14 & H3/M37 & Roots & 167 & LOC_Os05g 33400 & 66 & Basic 7S globulin precursor & - & - & - & - & + & + & + & + & - & - & - & - \\
\hline R15 & H5/M39 & Roots & 156 & Os10g0124000 & 92 & Ribosomal protein $\mathrm{L} 7 \mathrm{Ae}$ & + & + & + & + & - & - & - & - & + & + & + & + \\
\hline
\end{tabular}

“H”: Hpa II; "M": Msp I; “E”: EcoR I; “+”: Present; “-”: Absent 
A

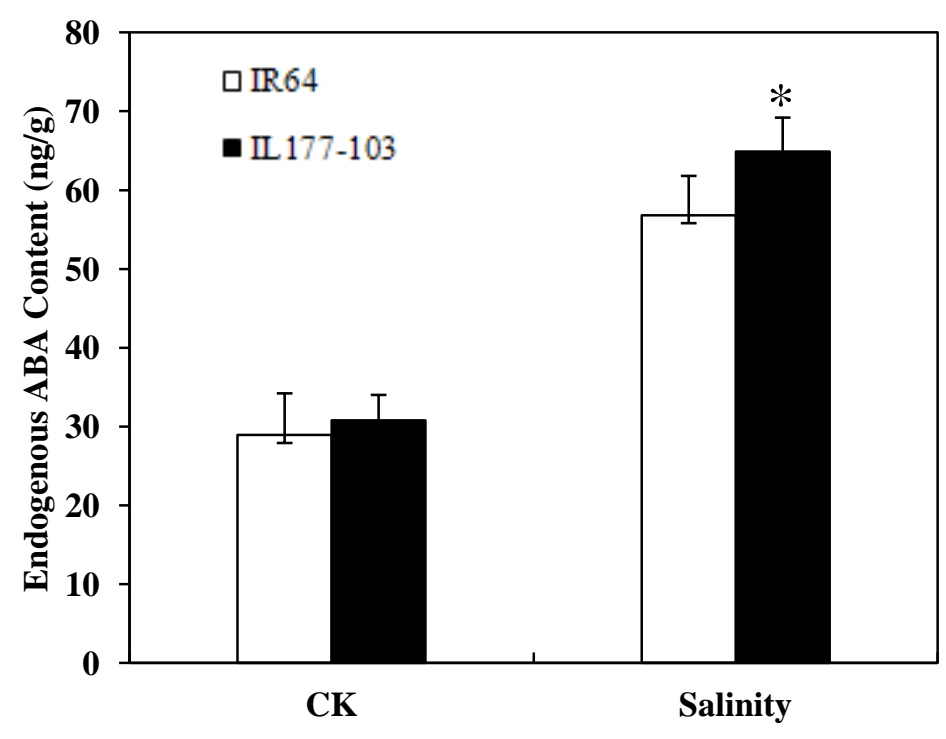

$\mathrm{C}$

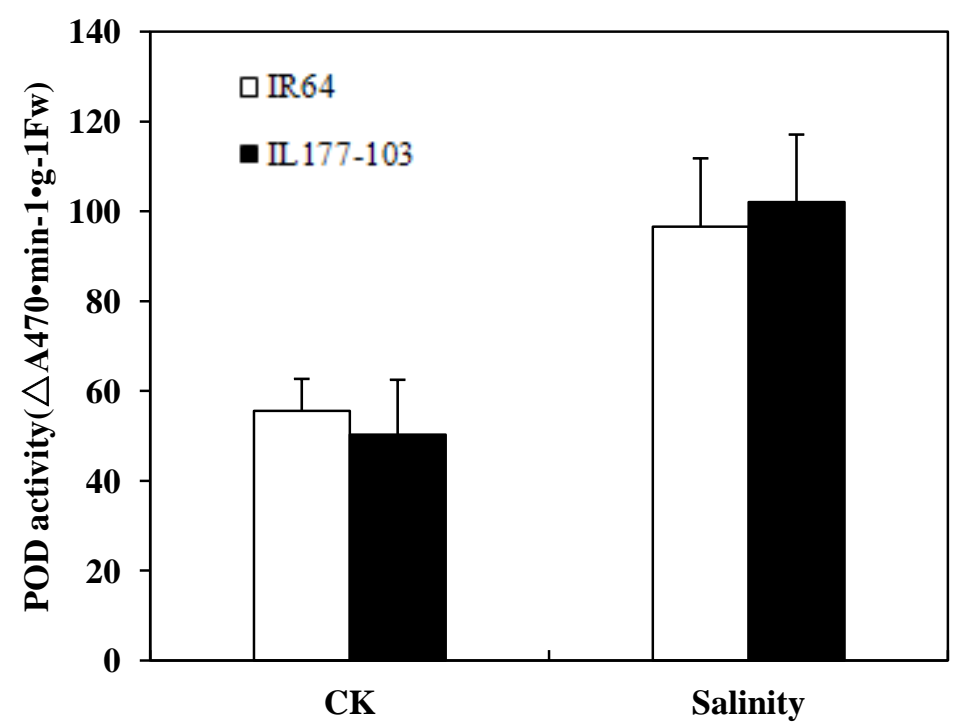

B



$\mathrm{D}$

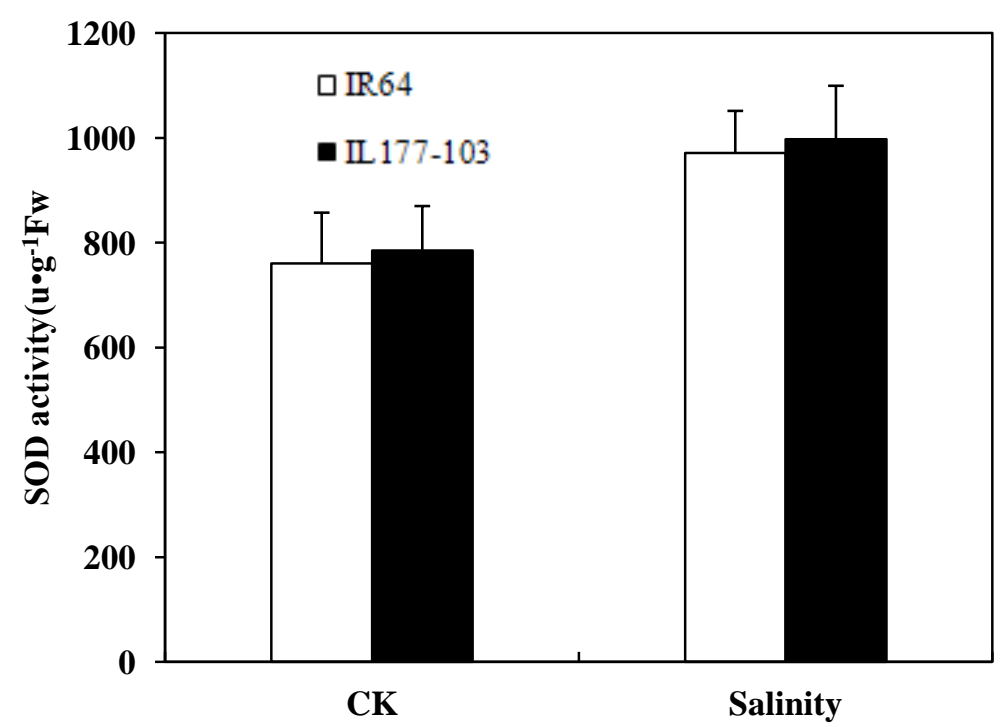

Figure 1. Endogenous ABA content and activity of antioxidant enzymes in seedlings of the two rice genotypes under control(CK) and salinity conditions. (A) Endogenous ABA content, (B) CAT activity, (C) POD activity, (D) SOD activity. IR64 cultivar (white) and IL177-103 cultivar (black). Values are means of 3 replicates. ${ }^{* *} \mathrm{p}<0.01 ;{ }^{*} \mathrm{p}<0.05$ versus IR64 (ANOVA, Dunnett's multiple comparison test). 